\title{
Corrigendum to
}

\section{"The relative roles of $\mathrm{CO}_{2}$ and palaeogeography in determining late Miocene climate: results from a terrestrial model-data comparison" published in Clim. Past, 8, 1257-1285, 2012}

\author{
C. D. Bradshaw ${ }^{1}$, D. J. Lunt ${ }^{1}$, R. Flecker ${ }^{1}$, U. Salzmann ${ }^{2}$, M. J. Pound ${ }^{2,3,4}$, A. M. Haywood ${ }^{3}$, and J. T. Eronen ${ }^{5,6}$ \\ ${ }^{1}$ Bristol Research Initiative for the Dynamic Global Environment (BRIDGE), School of Geographical Sciences, University of \\ Bristol, University Road, Bristol, BS8 1SS, UK \\ ${ }^{2}$ School of the Built and Natural Environment, Northumbria University, Newcastle upon Tyne, NE1 8ST, UK \\ ${ }^{3}$ School of Earth and Environment, University of Leeds, Leeds, LS2 9JT, UK \\ ${ }^{4}$ British Geological Survey, Kingsley Dunham Centre, Keyworth, Nottingham, NG12 5GG,UK \\ ${ }^{5}$ Department of Geosciences and Geography, P.O. Box 64, 00014, University of Helsinki, Finland \\ ${ }^{6}$ Biodiversity and Climate Research Centre LOEWE BiK-F, Senckenberganlage 25, 60325 Frankfurt am Main, Germany
}

Correspondence to: C. D. Bradshaw (c.bradshaw @bristol.ac.uk)

In the original manuscript, Figs. 7-16 included fonts which were not correctly embedded in the file. As such, unless certain propriety software (ArcGIS) is installed on the viewing platform, the figures will appear corrupted. In this Corrigendum, Figs. 7-16 and their captions are reproduced with the fonts correctly embedded.

Please find the correct figures below.

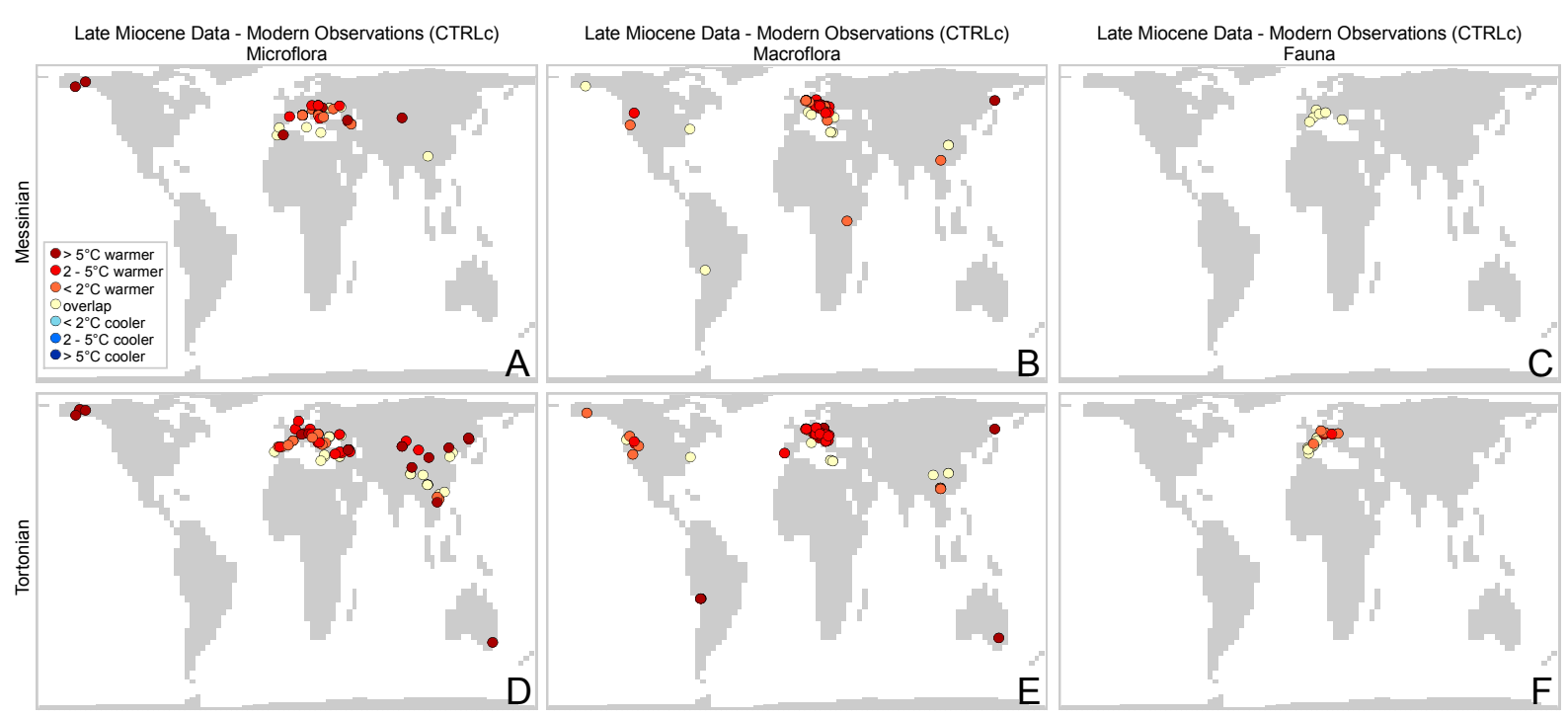

Fig. 7. Results from the model-data comparison for mean annual temperature, late Miocene data-modern potential natural climate estimates. 


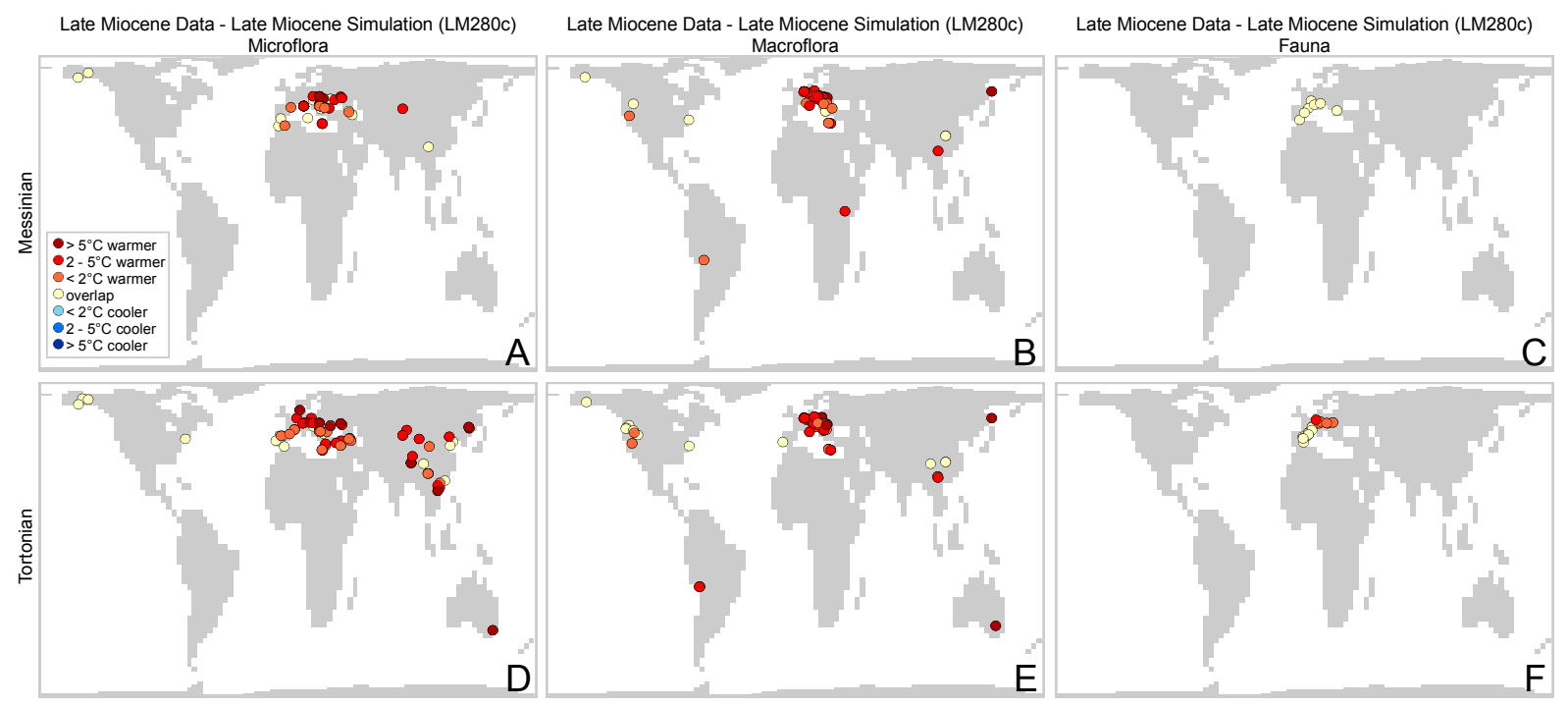

Fig. 8. Results from the model-data comparison for mean annual temperature, late Miocene data-LM280c.

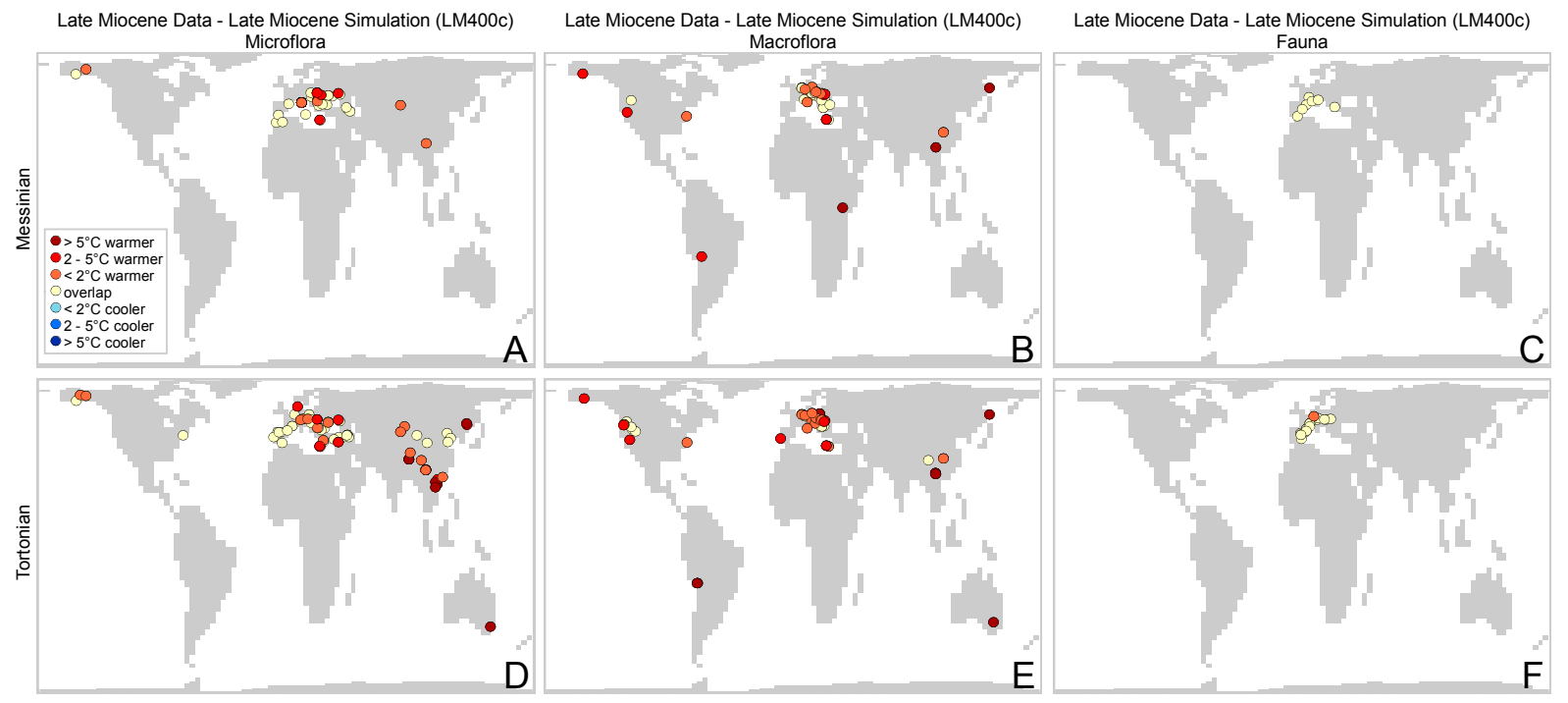

Fig. 9. Results from the model-data comparison for mean annual temperature, late Miocene data-LM400c. 


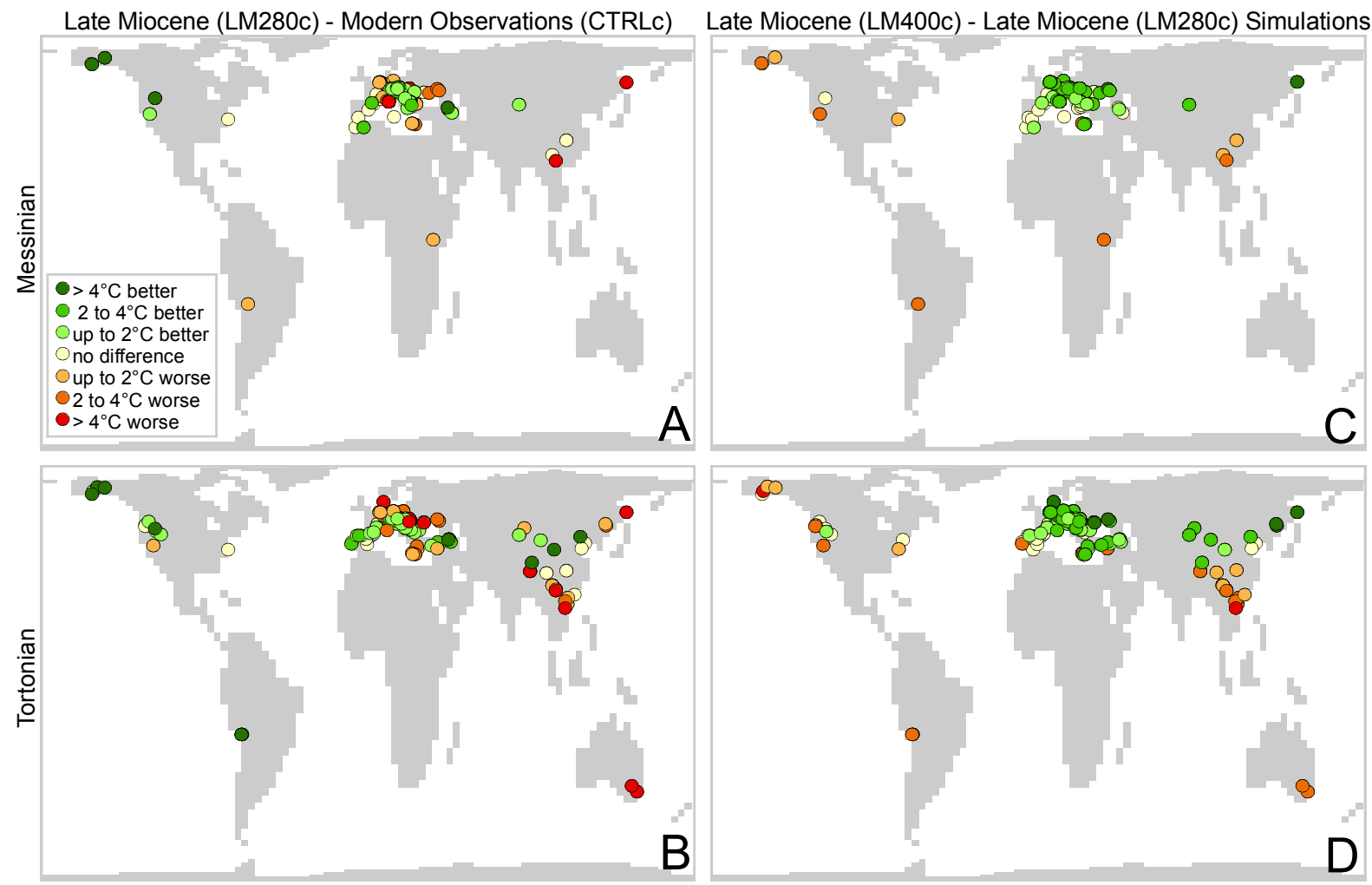

Fig. 10. Improvements in the model-data comparison for mean annual temperature. The lefthand column (A, B) shows the improvement that the late Miocene palaeogeography makes to the model-data comparison. The righthand column (C, D) shows the improvement that higher $\mathrm{CO}_{2}$ makes to the model-data comparison. Green circles indicate an improvement; red circles indicate a deterioration. The datapoints showing "no difference" are plotted underneath the other datapoints in order to highlight the differences.

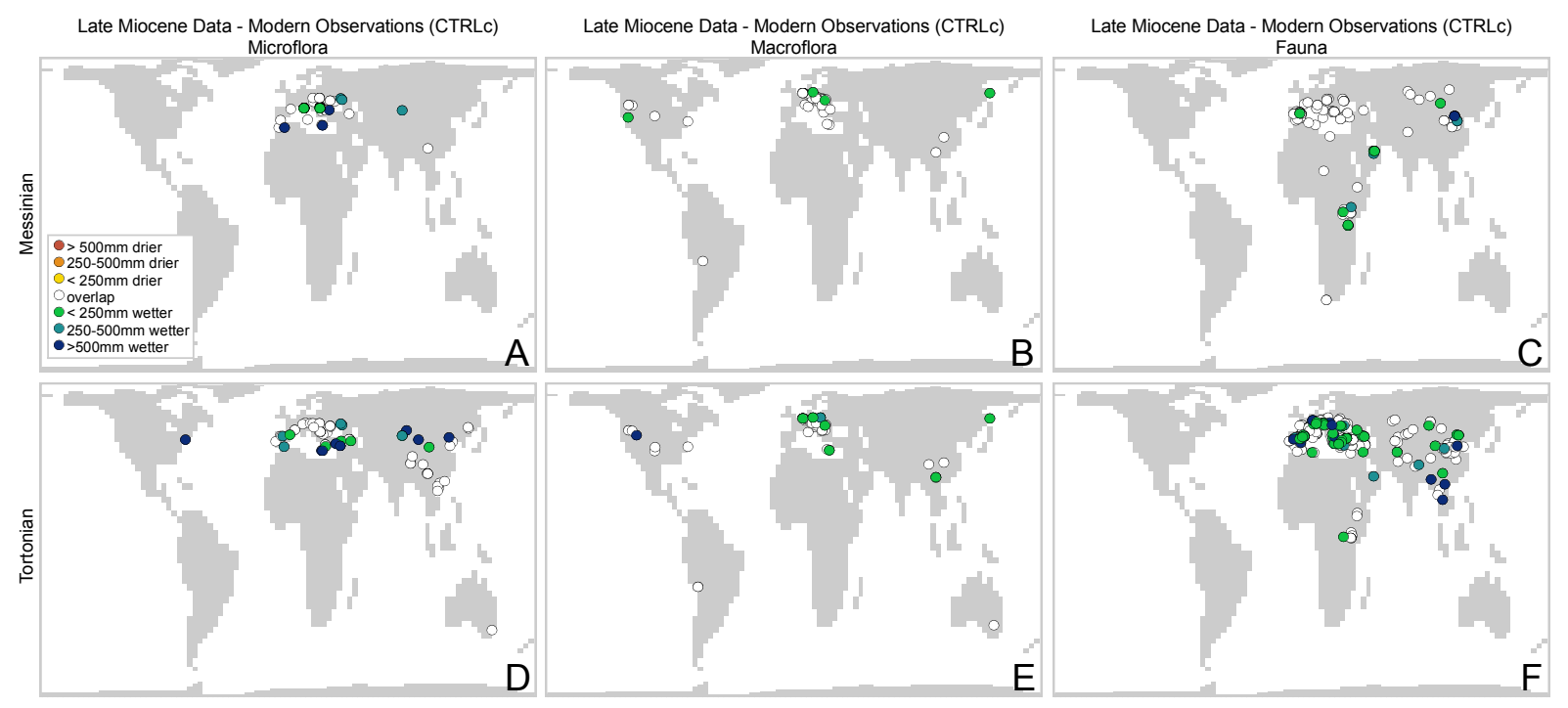

Fig. 11. Results from the model-data comparison for mean annual precipitation, late Miocene data-modern potential natural climate estimates. 


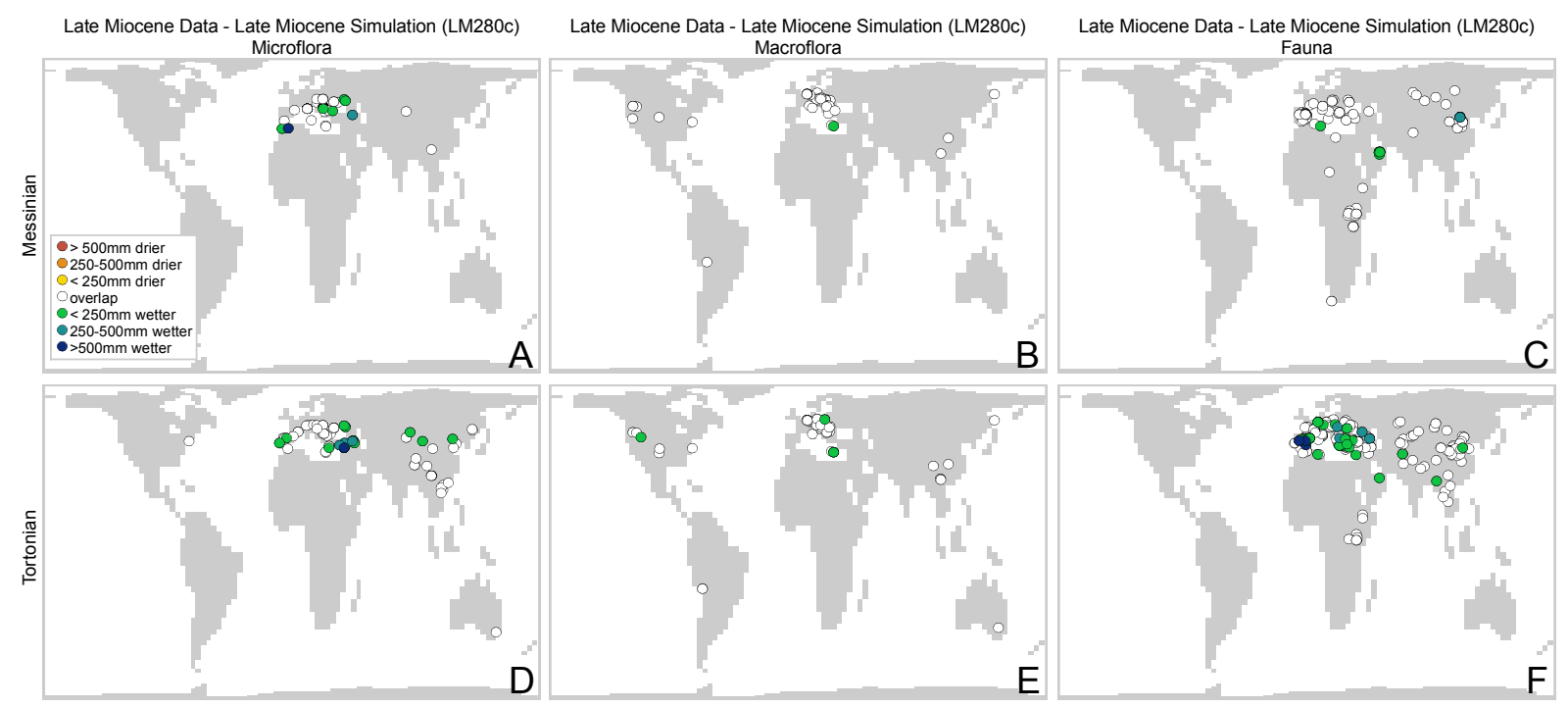

Fig. 12. Results from the model-data comparison for mean annual precipitation, late Miocene data-LM280c.

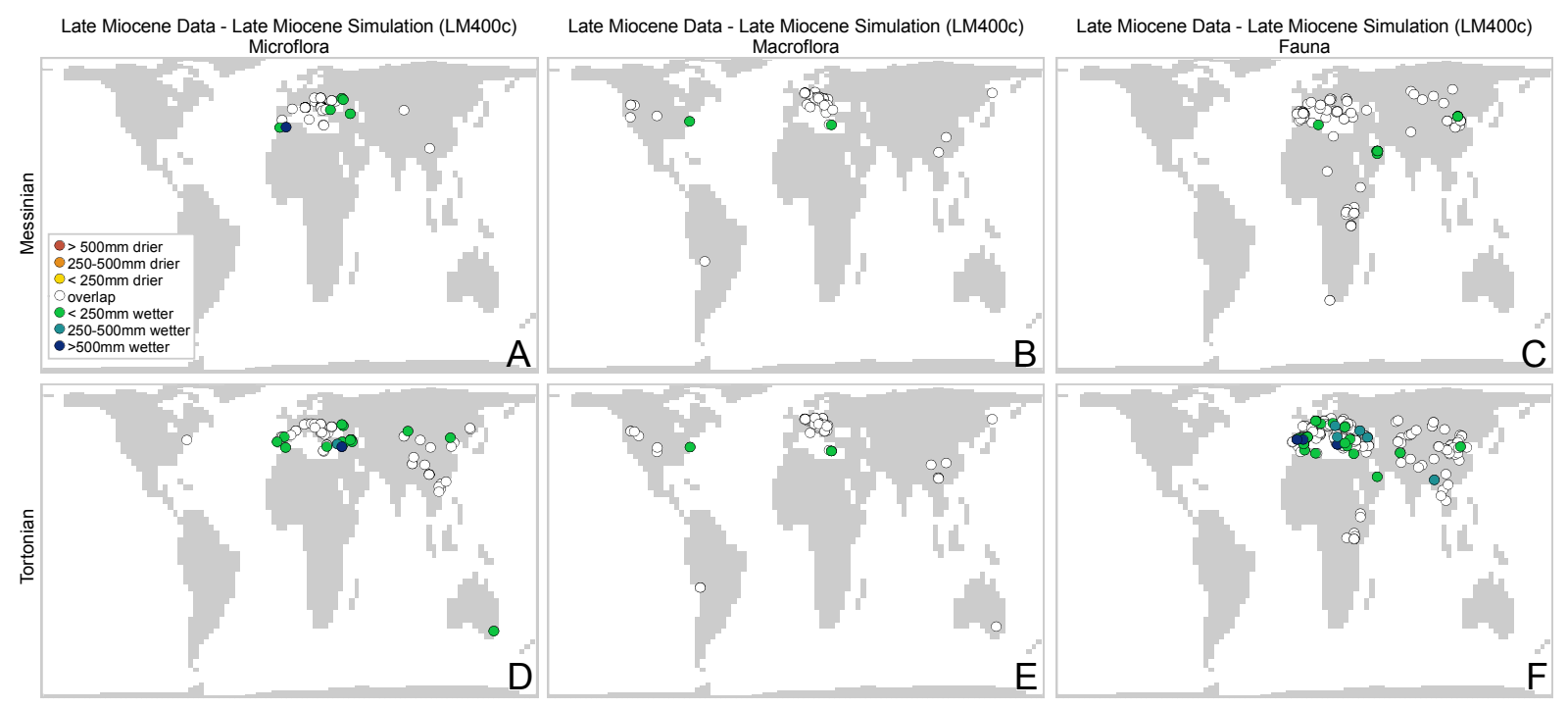

Fig. 13. Results from the model-data comparison for mean annual precipitation, late Miocene data-LM400c. 


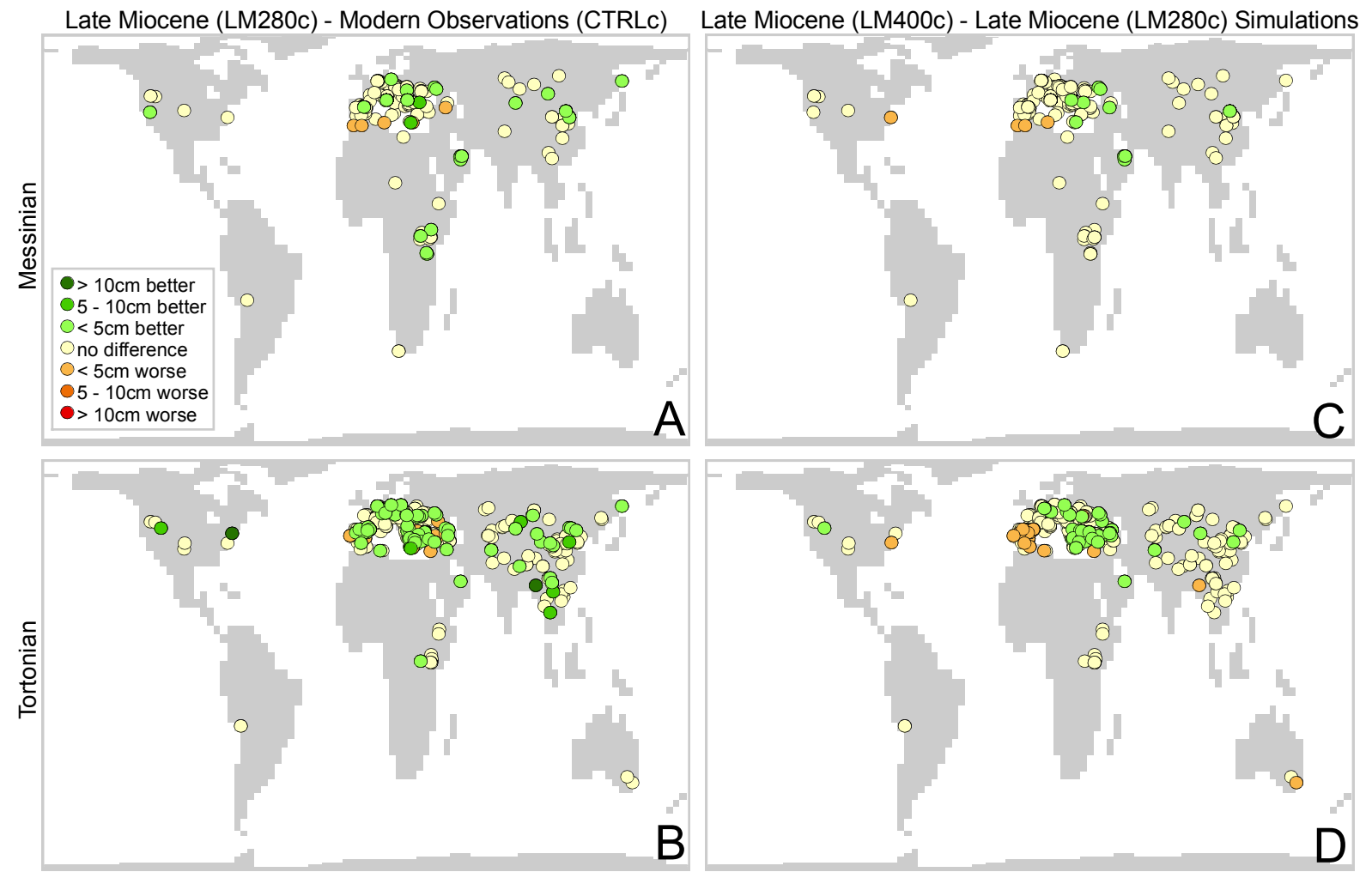

Fig. 14. Improvements in the model-data comparison for mean annual precipitation. The lefthand column (A, B) shows the improvement that the late Miocene palaeogeography makes to the model-data comparison. The righthand column $(\mathbf{C}, \mathbf{D})$ shows the improvement that higher $\mathrm{CO}_{2}$ makes to the model-data comparison. Green circles indicate an improvement; red circles indicate a deterioration. The datapoints showing "no difference" are plotted underneath the other datapoints in order to highlight the differences. 

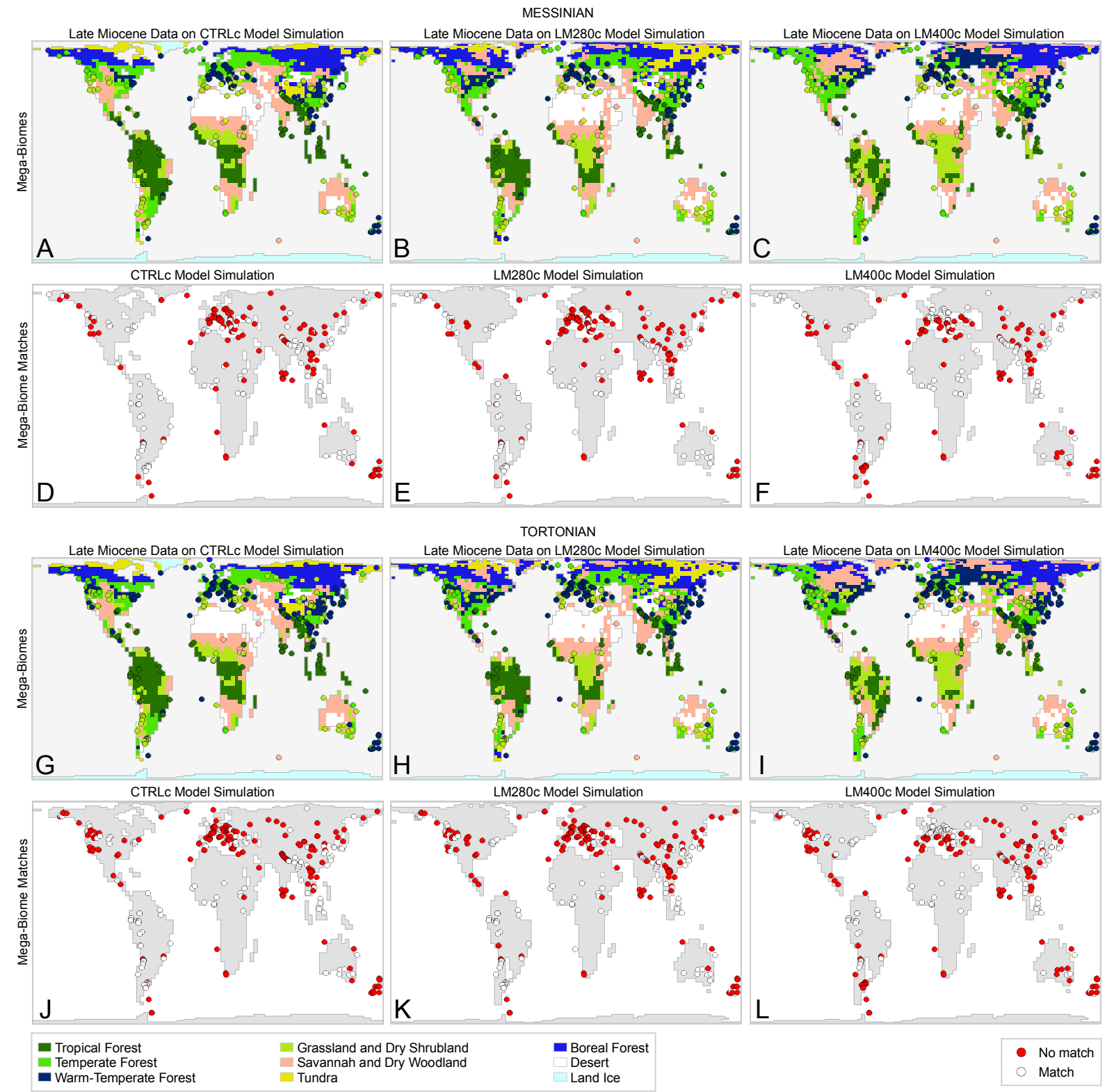

Fig. 15. Results from the model-data comparison for megabiomes: late Miocene data on CTRLc, LM280c, and LM400c. 

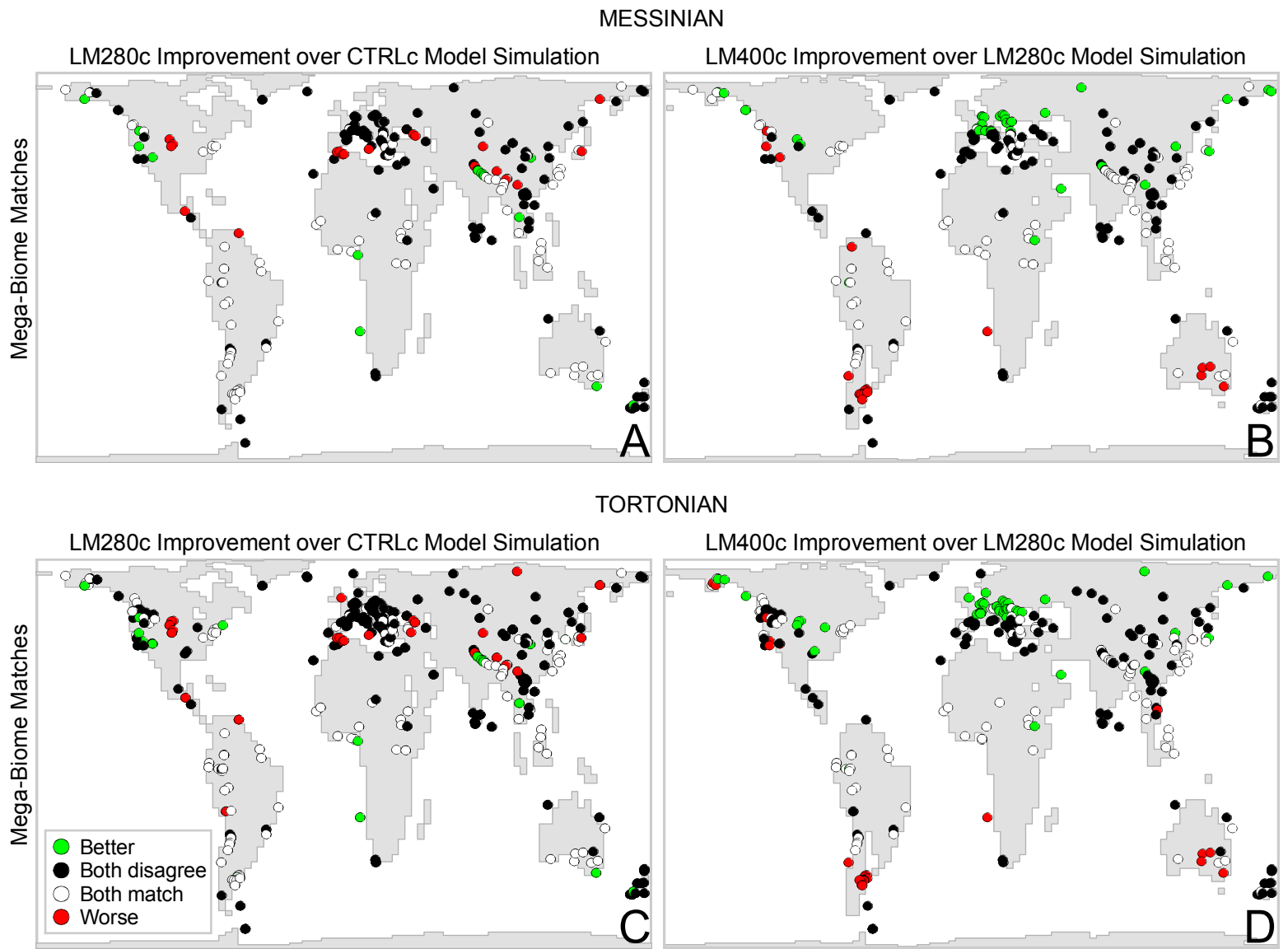

Fig. 16. Improvements in the model-data comparison for megabiomes. Panels (A) and (C) show the improvement that the late Miocene palaeogeography makes to the model-data comparison. Panel (B) and (D) show the improvement that higher $\mathrm{CO}_{2}$ makes to the model-data comparison. Green circles indicate an improvement; red circles indicate a deterioration. 